\title{
MicroRNAs in central nervous system disorders: current advances in pathogenesis and treatment
}

\author{
Mona Hussein ${ }^{1}$ and Rehab Magdy ${ }^{2^{*}}$ (D)
}

\begin{abstract}
MicroRNAs (miRNAs) are a class of short, non-coding, regulatory RNA molecules that function as post transcriptional regulators of gene expression. Altered expression of multiple miRNAs was found to be extensively involved in the pathogenesis of different neurological disorders including Alzheimer's disease, Parkinson's disease, stroke, epilepsy, multiple sclerosis, amyotrophic lateral sclerosis, and Huntington's disease. miRNAs are implicated in the pathogenesis of excitotoxicity, apoptosis, oxidative stress, inflammation, neurogenesis, angiogenesis, and bloodbrain barrier protection. Consequently, miRNAs can serve as biomarkers for different neurological disorders. In recent years, advances in the miRNA field led to identification of potentially novel prospects in the development of new therapies for incurable CNS disorders. MiRNA-based therapeutics include miRNA mimics and inhibitors that can decrease or increase the expression of target genes. Better understanding of the mechanisms by which miRNAs are implicated in the pathogenesis of neurological disorders may provide novel targets to researchers for innovative therapeutic strategies.
\end{abstract}

Keywords: MicroRNAs mimics and inhibitors, MicroRNAs-based therapeutics, Oxidative stress, Apoptosis, Neuroprotection

\section{Introduction}

MiRNAs are a class of short, non-coding RNA molecules that contain 19-24 nucleotides. They usually regulate gene expression at the messenger RNA (mRNA) level [1]. There is strong evidence that miRNAs have a role in different cellular processes including neural cells proliferation and differentiation, cell specification, cellular metabolism [1, 2].

miRNA are implicated in the pathogenesis of excitotoxicity, apoptosis, oxidative stress, inflammation, neurogenesis, angiogenesis, and blood-brain barrier protection [3]. Therefore, it is not surprising that miRNAs have emerged as key regulators of pathophysiology of different neurological disorders including Alzheimer's disease, Parkinson's

\footnotetext{
* Correspondence: rehab.m.hassan@kasralainy.edu.eg

${ }^{2}$ Lecturer of Neurology, Kasr Al-Ainy, Faculty of Medicine, Cairo University,

Cairo, Egypt

Full list of author information is available at the end of the article
}

disease, stroke, epilepsy, multiple sclerosis, amyotrophic lateral sclerosis, and Huntington's disease [4].

MiRNAs are released as circulating molecules into body fluids such as CSF, blood, and urine and therefore, they may be valuable biomarkers for detecting early onset neurodegenerative disorders. MiRNAs have the potential to be therapeutic molecules, where miRNA inhibitors and mimics can be used to target pathological upregulated and down-regulated miRNAs [5].

The objective of this review is to provide a brief synopsis about the role of miRNAs as key regulators and novel therapeutic targets in different neurological disorders including Alzheimer's disease, Parkinson's disease, stroke, epilepsy, multiple sclerosis, Amyotrophic lateral sclerosis, and Huntington's disease. 


\section{Main text}

\section{miRNA biogenesis}

MiRNA genes are transcribed by RNA polymerase II into primary miRNA (pri-miRNA) transcripts. The Drosha (a class 2 ribonuclease III enzyme) and DiGeorge syndrome critical region 8 (DGCR8, an RNA-binding protein) cleave the pri-miRNA into precursor miRNA (pre-miRNA). Exportin-5 binds to the pre-miRNA and helps their export into the cytoplasm. Dicer, also known as endoribonuclease Dicer, is an enzyme that cleaves the pre-miRNA into double strand RNA. One of the two strands of the miRNA duplex is incorporated into Argonaute (AGO) proteins to form the miRNA inducing silencing complex (miRISC) which leads to either translational repression or degradation of the target mRNA. The other miRNA strand is degraded [6].

\section{miRNA mimics and inhibitors}

MiRNA mimics are synthetic short double-stranded oligonucleotides imitating miRNA precursors. Once they are introduced into cells, they are recognized by miRNA biogenesis machinery and then processed accordingly [7]. MiRNA inhibitors (antagomirs) inhibit the interaction between miRNA and the miRISC or between the miRISC and its target mRNAs. They block the translation of mRNA into protein or induce its destruction. MiRNA-based therapeutics include miRNA mimics and inhibitors that can decrease or increase the expression of target genes [8].

\section{Mechanisms of miRNA-based therapeutics for neurological disorders Excitotoxicity}

Several miRNAs were found to attenuate excitotoxicity after ischemic stroke. Overexpression of miR-223 in hippocampal neurons protected them from neuronal death following transient global ischemia through decreasing the levels of NMDAR subunit $2 \mathrm{~B}$ and glutamate receptor 2 (GluR2) and halting NMDA-induced calcium influx [9]. MiR-181a inhibitor attenuated astrocyte dysfunction and hindered the decrease of glutamate transporter 1 resulting in enhancing the survival of hippocampal neurons [10].

\section{Apoptosis}

Some miRNA-based therapeutics were found to decrease apoptosis either by increasing the levels of anti-apoptotic proteins (Bcl-w, Bcl-2, Bcl-xl) or decreasing the levels of pro-apoptotic proteins (Puma, Noxa, Bax) [11]. miR-24, MiR-497, miR-15a/16-1, miR-181a, and miR-106b-5p antagomirs or miR-210 and miR-124 mimics were shown to attenuate the size of infarction in ischemic brain through increasing the levels of anti-apoptotic proteins (Bclw, Bcl-2, Bcl-xl) [12, 13]. A miR-124 inhibitor decreased infarction size in a mouse model through inhibiting apoptosis-stimulating proteins of p53 family [14]. MiR-23a, miR-21, miR-27a, and miR-23b decreased the levels of several pro-apoptotic proteins (Puma, Bax, Noxa, cleaved-caspase-3) in traumatic brain injury $[15,16]$. Activating miR-21, miR-20a, and miR-494 and inhibiting miR-29b attenuated apoptosis in spinal cord injury by activating AKT/ mTOR signaling pathway and inhibiting phosphatase and tensin homolog (PTEN) expression [17].

\section{Oxidative stress}

It has been demonstrated that downregulation of miR182 and miR-93, and upregulation of miR-424, miR-99a, and miR-23a-3p attenuated oxidative stress in ischemic brain [18-20]. miR-23a-3p mimic reduced oxidative stress in a mouse middle cerebral artery occlusion (MCAO) model through decreasing the production of 3nitrotyrosine (3-NT) and nitric oxide (NO) and increasing the expression of Manganese super oxide dismutase (SOD, a mitochondrial antioxidant enzyme that eliminates excess ROS) [21]. MiR-93 antagomir reduced infarction size and improved function outcome after ischemic stroke, via increasing the expression level of erythroid 2-related factor (Nrf2) and its downstream gene hemeoxygenase-1 (HO-1) [19]. In addition, miR486 inhibition ameliorated ROS in spinal cord injury through increasing the expression of NeuroD6, upregulation of glutathione peroxidase 3 and thioredoxin-like 1 [22].

\section{Inflammation}

Many miRNA-based therapeutics have antiinflammatory effect. Their anti-inflammatory actions involve the suppression of cytokines secretion, astrocytes activation, and leukocyte extravasation. Inhibition of $\mathrm{miR}-15 \mathrm{a} / 16-1$ or overexpression of miR-122 and miR-22 decreased the levels of the following inflammatory cytokines: TNF-a, IL-6, COX-2, VCAM1 , and iNOS in ischemic brain [23, 24]. Exosomemediated delivery of miR-124-3p reduced tissue inflammation and induced M2 microglia polarization after traumatic brain injury. The function of M2 microglia is to downregulate the inflammatory pathway, thus promoting tissue repair [24].

MiR-27a, miR-124, miR-199b, miR-133b, and miR497 mimics attenuated inflammatory responses in spinal cord injury through inhibition of NF-kB/IL-1b pathway and reduction of astrocyte/macrophage activation $[25,26]$. 


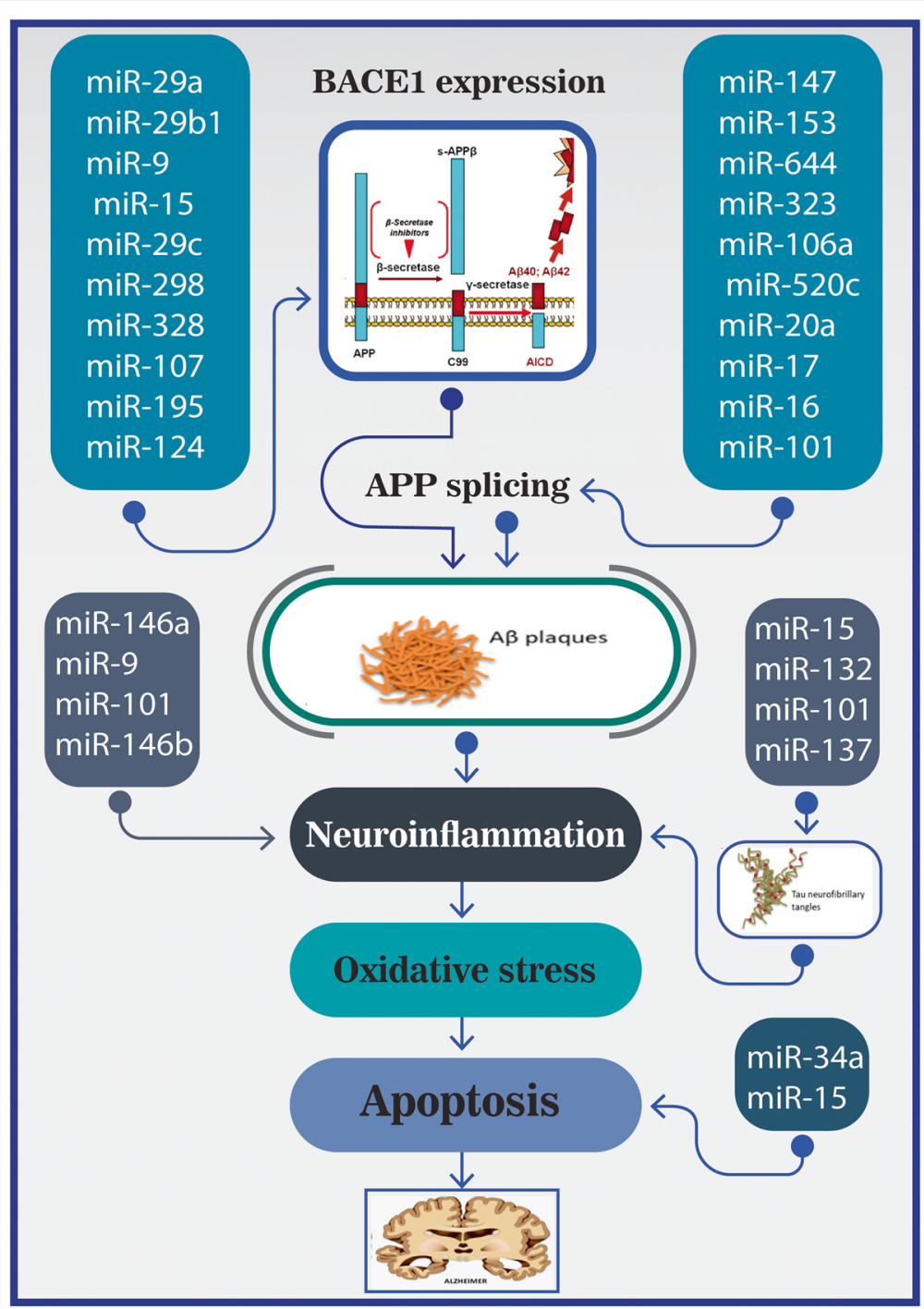

Fig. 1 Micro RNAs dysregulation in Alzheimer disease. Multiple micro RNAs are involved in different pathogenic mechanisms of Alzheimer's disease (modulating the activity of $\beta$-secretases, APP splicing, neurofibrillary tangles, neuroinflammation, or apoptosis). APP: amyloid precursor protein, BACE1: $\beta$-site APP cleaving enzyme 1

\section{Neurogenesis}

miRNAs were found to modulate neurogenesis in cerebral cortex and spinal cord in stroke, spinal cord injury and traumatic brain injury. Overexpression of the miR17-92 cluster in the subventricular zone (SVZ) significantly enhanced neurogenesis and promoted the proliferation of neural stem cells after acute ischemic stroke [27]. miR-20a inhibitor increased neurogenesis and enhanced the survival of motor neurons in mice following spinal cord injury through increasing expression of the miR-20a target gene neurogenin 1 [28].

\section{Angiogenesis}

Several miRNAs were found to modulate angiogenesis in stroke, spinal cord injury and traumatic brain injury.
MiR-107 mimic increased the number of capillaries in penumbra and reduced infarction size in ischemic brain through increasing the levels endothelial VEGF165/ 164 [29]. MiR-21 mimic improved functional outcome following traumatic brain injury via upregulation of Angiopoietin-1 (Ang-1), Tie-2 (receptor of Ang-1), and VEGF [30]. MiR-210 and mir-223 enhanced angiogenesis in spinal cord injury [31, 32].

\section{Blood brain barrier/blood spinal cord barrier (BBB/BSCB) protection}

The inflammatory cascade following damage to the $\mathrm{BBB}$ or the BSCB can be modulated by some miRNAs. Downregulating miR-150 alleviated BBB disruption after ischemic stroke through increasing claudin-5 and 


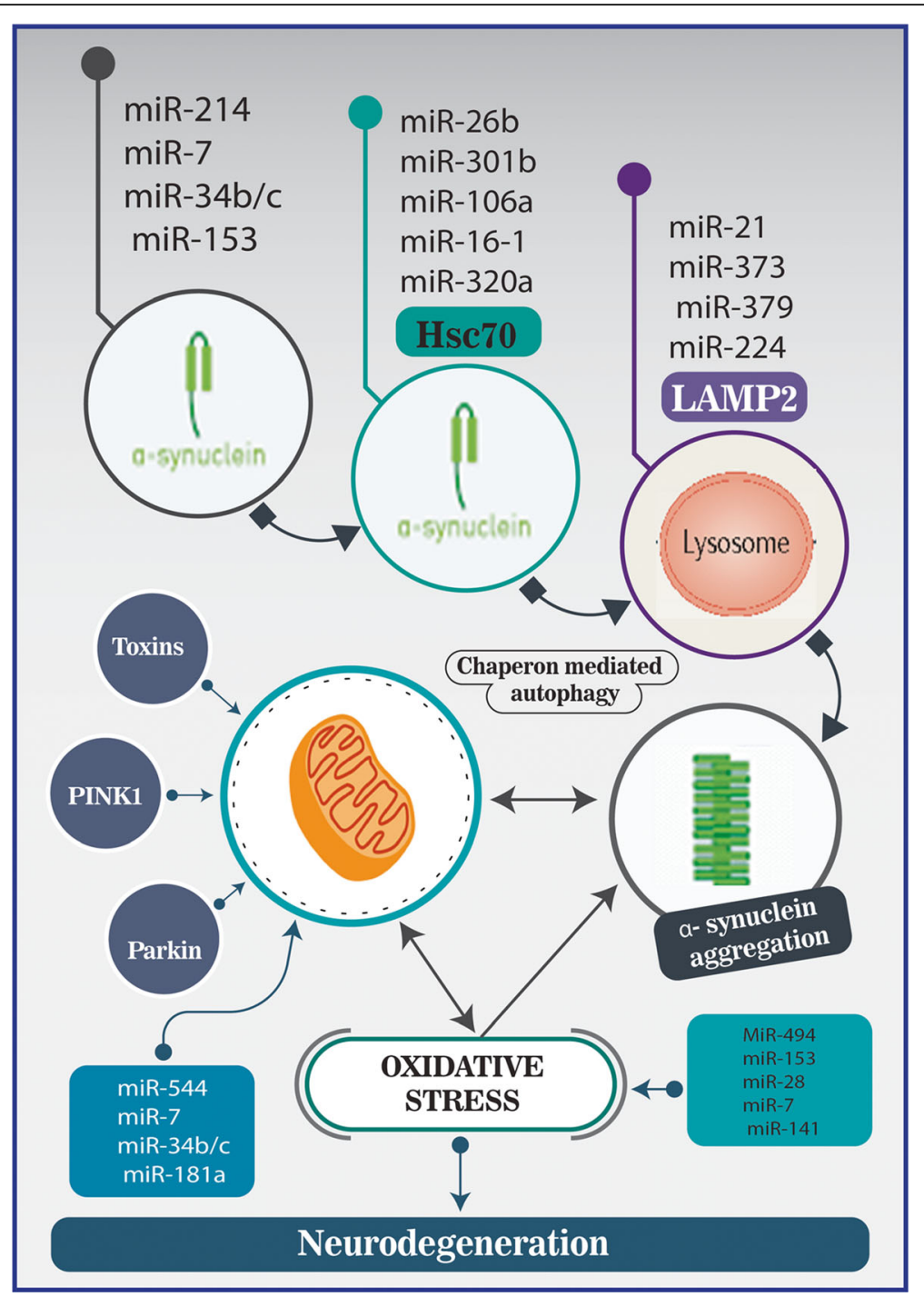

Fig. 2 Micro RNAs dysregulation in Parkinson disease. Multiple microRNAs were reported to control a-synuclein aggregation either by direct regulation or by chaperon-mediated autophagy. Others control mitochondrial function or oxidative stress. LAMP2A: Lysosomal-associated membrane protein 2A, Hsp70: heat shock protein 70

stabilization of TJ protein ZO-1,191. Anti-mir-130 and anti-miR-320 upregulated the expression of aquaporins following ischemic stroke, which are implicated in the clearance of cerebral edema [33, 34]. MiR-320a mimic attenuated BBB disruption, ameliorated spinal cord reperfusion, and decreased water content in the spinal cord through suppressing the expression of AQP1 [35].

\section{MiRNAs dysregulation in neurological disorders MiRNAs dysregulation in Alzheimer's disease}

Altered expression of some miRNA in patients suffering from $\mathrm{AD}$ suggests that miRNA may have a crucial regulatory role on the mechanisms involved in the pathogenesis of $\mathrm{AD}$, including beta-amyloid $(\mathrm{A} \beta)$ metabolism by modulating the activity of $\beta$-secretases such as BACE1 and tau aggregation leading to neurofibrillary tangle (NTF) formation (Fig. 1) [36, 37].

\section{MiRNA dysregulation in Parkinson's disease}

Multiple microRNAs were reported to control $\alpha$ synuclein aggregation either by direct regulation, or by chaperon-mediated autophagy and their downregulation may contribute to $\alpha$-synuclein-mediated neurotoxicity in PD [38-40], (Fig. 2). In PD, there was upregulation of some of these microRNAs [41]. Some microRNAs were reported to be implicated in neuroinflammation, such as miR-124 and miR-146a (anti-inflammatory) and miR155 (pro-inflammatory) [42]. MiR-124 enhanced the survival of dopaminergic neurons and attenuated microglial activation in MPTP model of PD [43]. 


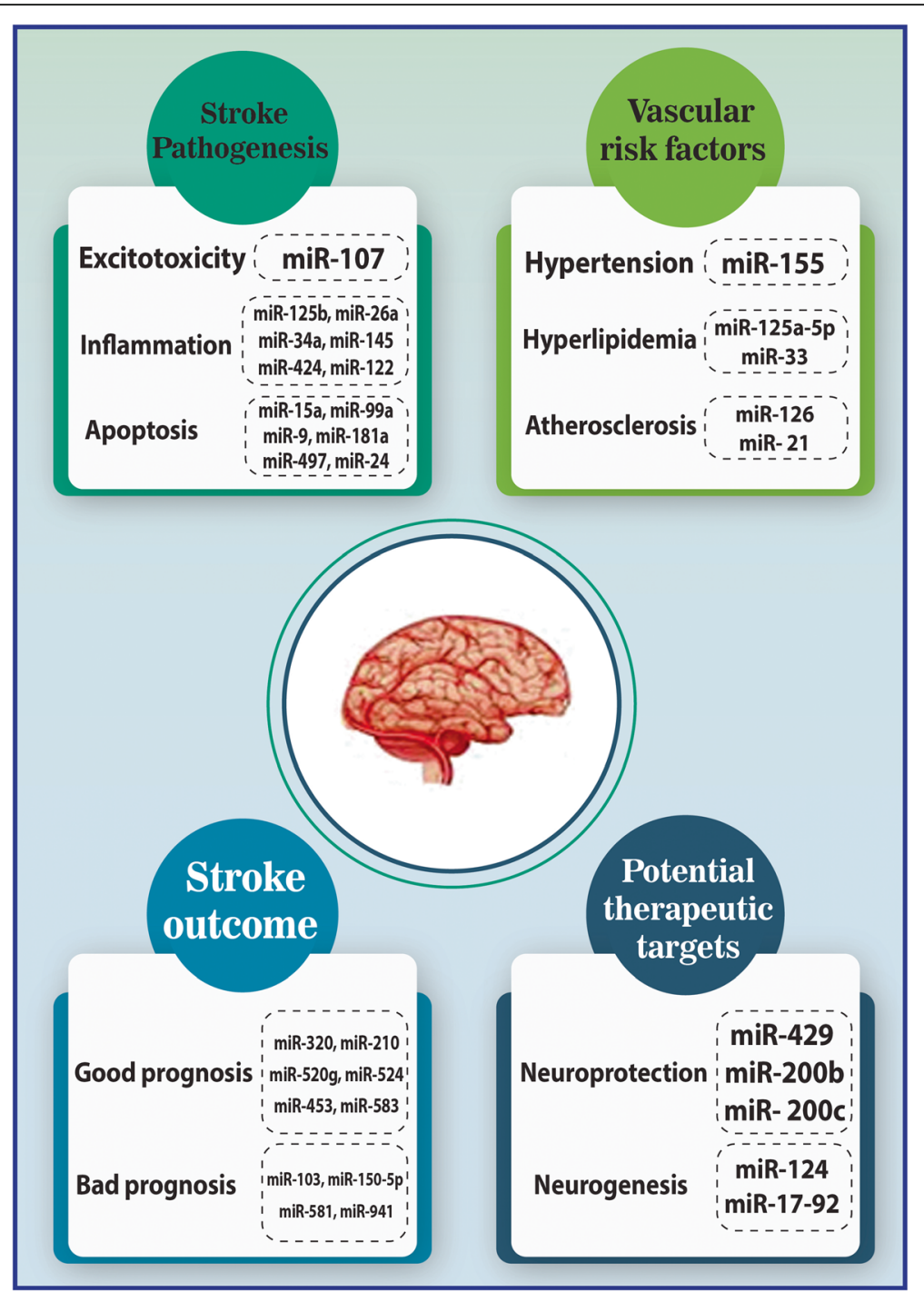

Fig. 3 Micro RNAs dysregulation in ischemic stroke. Multiple microRNAs in pathogenic mechanisms (excitotoxicity, inflammation, and apoptosis), risk factors, and stroke outcome. Some are promising therapeutic targets (neurogenesis and neuroprotection)

miRNAs dysregulation in ischemic stroke

Several reports demonstrated that miRNAs have distinct expression patterns that modulate pathophysiological process of stroke [44]. Tan and colleagues carried out miRNA profiling from blood of young patients with stroke and identified characteristic patterns in ischemic stroke [45]. Moreover, anti-miR-320a led to a reduction of infarct size in ischemic stroke with a concomitant increase in aquaporins- 1 and 4 mRNA [33]. After subjecting rat brains to MCAO then reperfusion for 24 or $48 \mathrm{~h}$, 114 miRNAs were detected in ischemic brain samples. Among them, 82 and 106 transcripts were detected in the 24-h and 48-h reperfusion [46].

The pattern of miRNA profile in patients with small artery stroke is distinctly different from that of large artery stroke. Therefore, microRNA profiling can be used to predict the stroke subtypes [47]. Some miRNAs can be used as novel biomarkers for diagnosis and prognosis in acute ischemic stroke, and some were investigated as potential therapeutic targets [47, 48], (Fig. 3).

\section{MiRNAs dysregulation in epilepsy}

There is strong evidence that miRNAs dysregulation were linked to the mechanisms of epileptogenesis through regulating ion channels, inflammatory response, synaptic plasticity, and neuronal apoptosis. Some miRNAs were reported to influences molecular and cellular pathways implicated in epilepsy, including oxidative stress, inflammation, immune responses, cell differentiation, migration, and proliferation [49-51] (Fig. 4). 


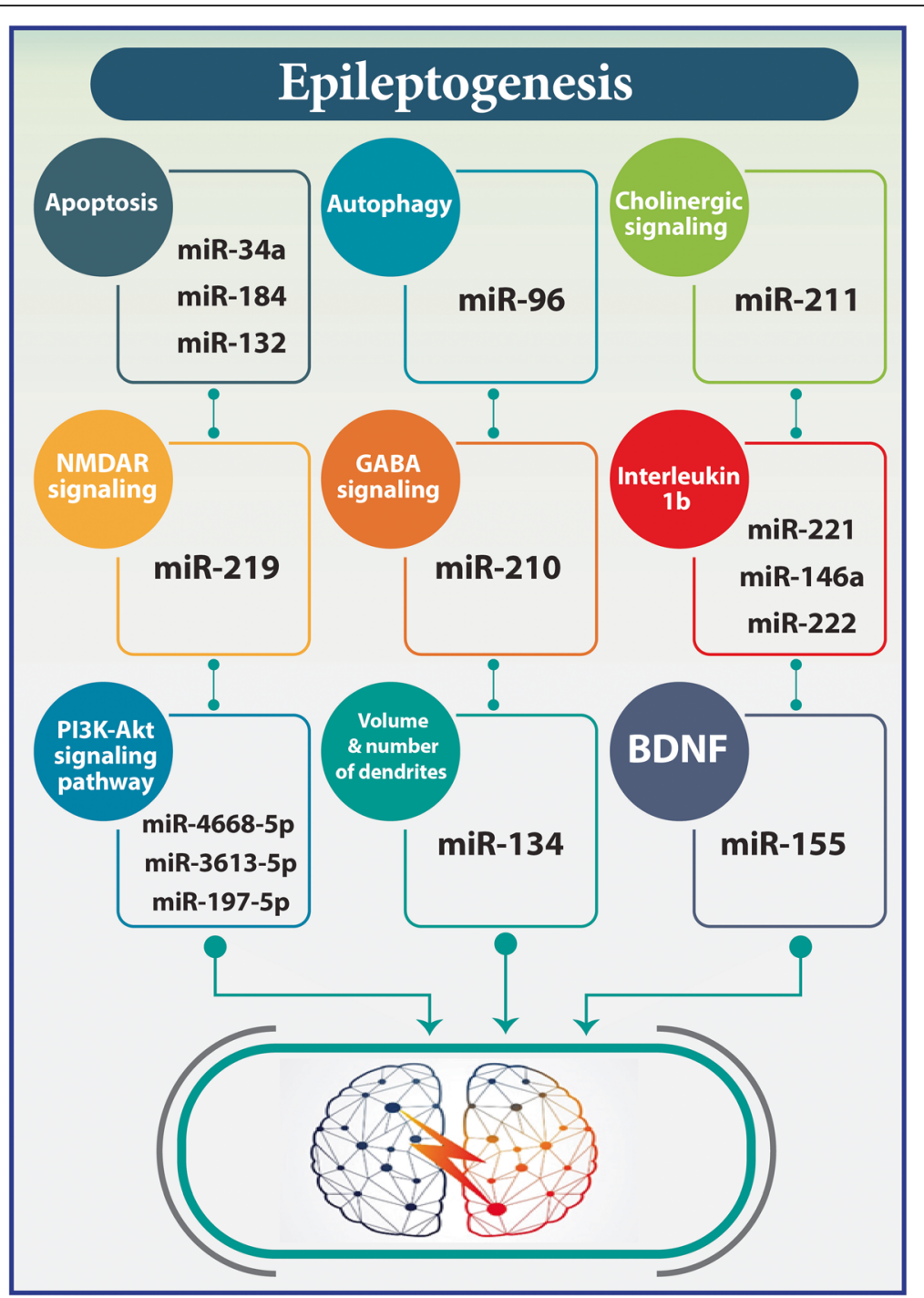

Fig. 4 Micro RNAs dysregulation in epileptogenesis. miRNAs dysregulation were linked to the mechanisms of epileptogenesis through regulating ion channels, neuroinflammation, synaptic plasticity, and neuronal apoptosis. GABA: $\gamma$-aminobutyric acid, NMDA: N-methyl-d-aspartate, PI3K-Akt: The phosphatidylinositol 3-kinase/protein kinase B, BDNF: Brain-derived neurotrophic factor

Targeting these miRNAs is a challenge for future strategies for anti-epileptogenesis therapy.

Yuan, Huang [52] found that silencing miR-132 had a neuroprotective effect on epileptic mouse models through regulating the morphology and electrophysiology of dendritic spines. The expression of miR-22, miR-34a, miR-21, and miR-125a in blood and the hippocampus were found to be changed 24 hours after the onset of status epilepticus [53].

\section{MiRNA dysregulation multiple sclerosis}

MiRNAs dysregulation display strong association with multiple sclerosis (MS). Several MiRNAs were reported to be consistently upregulated in MS patients including miR-142-3p, miR-145, miR-146a/b, miR-22,
miR-155, miR223/-3p, miR-584, and miR-326. Overexpression of these miRNAs in MS patients suggests their implication in the pathogenic inflammatory process observed in MS. miR-155 was one of the most consistently dysregulated miRNA in MS. It has a role in disruption of the blood-brain barrier, immune cell activation and neurodegeneration [54, 55]. Upregulation of miR-155 was significantly reduced by immunomodulatory medications such as glatiramer acetate (GA), supporting its potential role in the pathogenic pro-inflammatory process [56, 57]. miR146 has also been reported to be upregulated in the blood and CNS lesions of MS patients [57].

miR-17, miR-21, miR-320, and miR-150 exhibited different patterns across the compartments in MS patients 


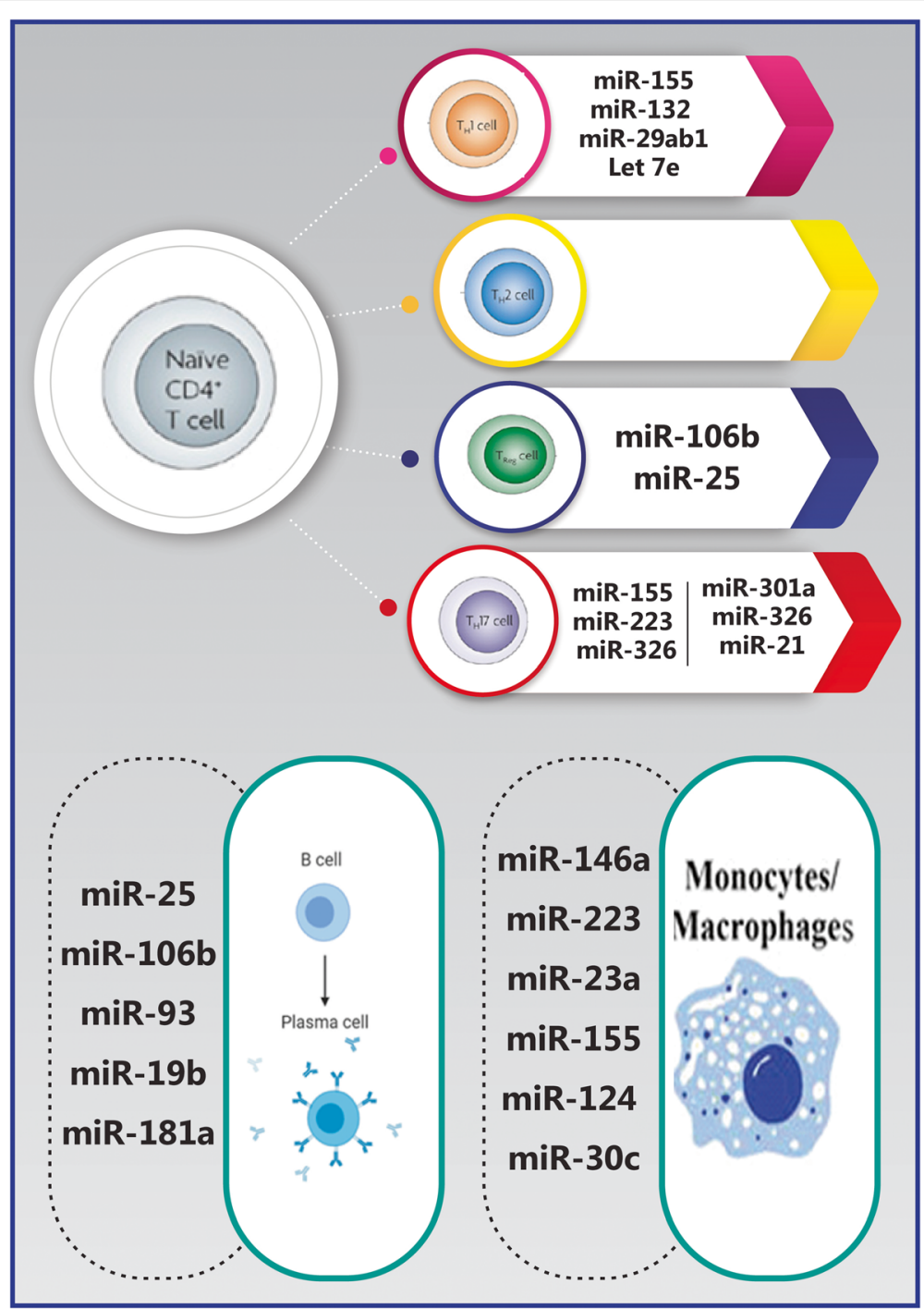

Fig. 5 Micro RNAs dysregulation in immune compartment of multiple sclerosis. Dysregulation of different MiRNAs were reported to control the inflammatory activity of various immune cells (Th1, Treg, Th17, macrophages, and B cells)

with predominant upregulation in the CNS lesions and downregulation in the immune tissue $[55,58,59]$. Interestingly, It has been reported that miR-21 exhibit both pro- and anti-inflammatory functions. It is upregulated in the active disease and downregulated in remission state and in secondary progressive MS [60].

On the other hand, members of the miR-103, miR-548 miR-15, and let-7 families were consistently downregulated in MS patients. Let-7 and miR-548 family members were exclusively dysregulated in the immune compartment while miR-103 family and miR-15a/b were downregulated in all cellular compartments apart from regulatory $\mathrm{T}$ cells [55]. Dysregulation of different MiRNAs targeting the inflammatory activity of various immune cells was shown in Fig. 5 [55, 58, 61, 62].

\section{MiRNA dysregulation in Amyotrophic lateral sclerosis}

Multiple differentially expressed miRNAs are implicated in the pathophysiology of ALS. miR-27a, miR-34a, miR155, miR-142-5p, and miR-338-3p were indicated as novel biomarkers and potential therapeutic targets in ALS [63, 64].

MiR-155 is upregulated in both sporadic and familial ALS patients, and inhibiting it in the brains of SOD1G93A mice model increases survival [63]. miR34a is dysregulated in ALS. It regulates X-linked inhibitor of apoptosis (XIAP) and Sirtuin 1 (SIRT1), which is protective against oxidative stress-induced apoptosis [65, 66]. ALS patients were also found to have reduced expression of miR-34a, which is rescued by treatment with enoxacin [65]. Thus, microRNA 


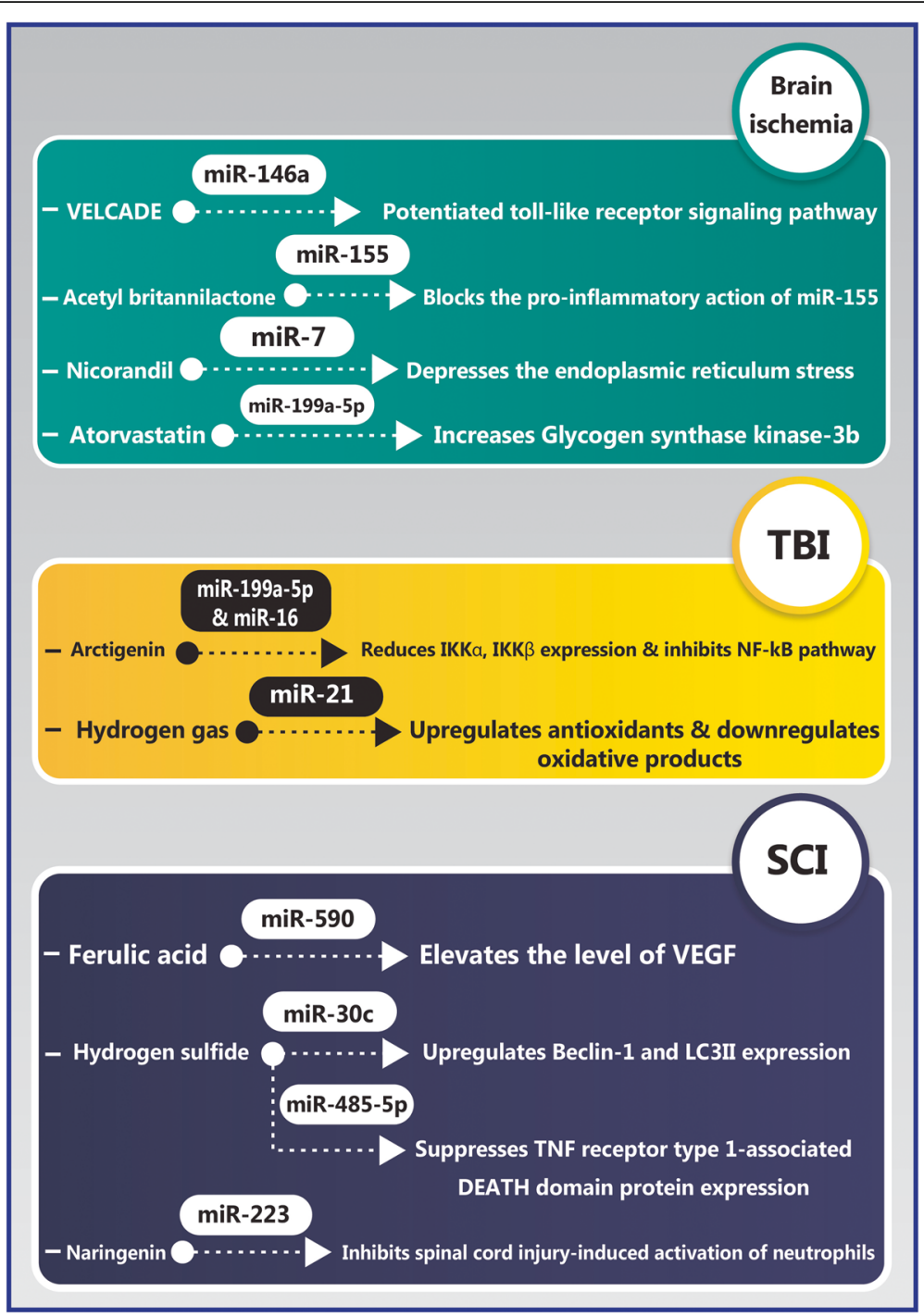

Fig. 6 Micro RNAs-based therapeutics in different neurological disorders. Some pharmacological agents can exert neuroprotection through the regulation of functional miRNAs in ischemic stroke, traumatic brain injury, and spinal cord injury. TBl: traumatic brain injury, NF-Kb: nuclear factor kappa B, SCl: spinal cord injury, VEGF: vascular endothelial growth factor, TNF: tumor necrosis factor

biogenesis stimulating drugs can be potentially used in treatment of ALS [67].

\section{MiRNAs dysregulation in Huntington's disease (HD)}

Several reports indicated as strong association between miRNA and pathogenic mechanisms in HD. There is strong evidence that both transcription and processing of microRNAs appear to be dysregulated in HD [68]. Furthermore, microRNA sequencing and differential expression analysis demonstrated downregulation of miR29b, miR-124a, miR-9, and miR-9*. The two later are mature miRNAs that are produced by a single miR-9 precursor. Whereas miR-29a, miR-132, and miR-330 in the brains of patients with HD were upregulated [69, 70]. Analysis of HD mouse models also identified downregulation of miR-22, miR-128, miR-29c, miR-138, miR-132, miR-218; and miR-674, miR-344, and miR-222 [71].

\section{MiRNA-based therapeutics}

miRNA-based therapeutics include the pharmacological agents that exert neuroprotection through the regulation of functional miRNAs. Hydrogen gas was found to regulate oxidative stress via upregulating miR-21 [72]. VELCADE or bortezomib, which is used for treatment of multiple myelomas and mantle cell lymphoma, exerts neuroprotective effect against cerebral ischemia through upregulation of miR-146a [73]. Ferulic acid improves functional recovery in spinal cord injury by inhibiting miR-590 [74]. Additionally, 
there are other pharmacological agents including trimetazidine (TMZ) [75], acetylbritannilactone (ABL) [76], hydrogen sulfide (H2S) [77], and nicorandil [78] that depend on the modulation of specific miRNAs. So, blocking the modulation of these miRNAs can completely abolish the neuroprotective effects of these agents against CNS injuries (Fig. 6).

\section{Conclusion}

There is strong evidence that clearly demonstrates the association between miRNA dysregulation and neurodegenerative diseases. miRNA-based therapeutics have become one of the most promising strategies in treatment of incurable neurological disorders. Further researches are needed to identify candidate miRNAs, clarify how they exert their effects, design pharmacological formulations and delivery methods that can cross the BBB to target brain tissue, and develop methods to decrease off target effects.

\section{Abbreviations}

miRNAs: MicroRNAs; mRNA: Messenger RNA; DGCR8: DiGeorge syndrome critical region 8; UTR: Untranslatedregion; pri-miRNA: Primary miRNA; premiRNA: Precursor miRNA; AGO: Argonaute; miRISC: MiRNA inducing silencing complex; GluR2: Glutamate receptor 2; 3-NT: 3-nitrotyrosine; NO: Nitric oxide; HO-1: Hemeoxygenase-1; SVZ: Subventricular zone; BBB: Blood-brain barrier; BSCB: Blood spinal cord barrier; NFTs: Neurofibrillary tangles; MCAO: Middle cerebral artery occlusion; AD: Alzheimer's disease; APP: Amyloid precursor protein; BACE1: $\beta$-site APP cleaving enzyme 1; PD: Parkinson disease; LAMP2A: Lysosomal-associated membrane protein 2A; Hsp70: Heat shock protein 70; GABA: $\gamma$-aminobutyric acid; NMDA: N-methyl-d-aspartate; PI3KAkt: The phosphatidylinositol 3-kinase/protein kinase B; BDNF: Brain-derived neurotrophic factor; MS: Multiple sclerosis; GA: Glatiramer acetate; XIAP: Xlinked inhibitor of apoptosis; SIRT1: Sirtuin 1; ALS: Amyotrophic lateral sclerosis; TARDBP: TAR DNA-binding protein 43; SOD: Superoxide dismutase; HD: Huntington's disease; TBI: Traumatic brain injury; NF-Kb: Nuclear factor kappa B; SCI: Spinal cord injury; VEGF: Vascular endothelial growth factor; TNF: Tumor necrosis factor; TMZ: Trimetazidine; ABL: Acetylbritannilactone; H2S: Hydrogen sulfide

\section{Acknowledgements}

Not applicable

\section{Authors' contributions}

$\mathrm{MH}$ performed the major role in literature search and helped to draft manuscript. RM shared in the literature search and helped to draft manuscript. All authors have read and approved the manuscript.

\section{Funding}

Not applicable

\section{Availability of data and materials}

Not applicable

Ethics approval and consent to participate

Not applicable

\section{Consent for publication}

Not applicable

\section{Competing interests}

The authors declare that they have no competing interests.

\section{Author details}

'Associate Professor of Neurology, Beni-suef University, Beni-suef, Egypt.

${ }^{2}$ Lecturer of Neurology, Kasr Al-Ainy, Faculty of Medicine, Cairo University, Cairo, Egypt.

Received: 22 December 2020 Accepted: 15 February 2021

Published online: 04 March 2021

\section{References}

1. Guarnieri DJ, DiLeone RJ. MicroRNAs: a new class of gene regulators. Ann Med. 2008:40(3):197-208

2. Hatfield S, Ruohola-Baker H. microRNA and stem cell function. Cell Tissue Res. 2008:331(1):57-66.

3. McNeill E, Van Vactor D. MicroRNAs shape the neuronal landscape. Neuron. 2012;75(3):363-79.

4. Maciotta Rolandin S, Meregalli M, Torrente $\mathrm{Y}$. The involvement of microRNAs in neurodegenerative diseases. Front Cell Neurosci. 2013;7:265.

5. Rajgor D. Macro roles for microRNAs in neurodegenerative diseases. Noncoding RNA Res. 2018;3(3):154-9.

6. Winter J, Jung S, Keller S, Gregory Rl, Diederichs S. Many roads to maturity: microRNA biogenesis pathways and their regulation. Nat Cell Biol. 2009; 11(3):228-34.

7. Latronico MV, Condorelli G. Therapeutic use of microRNAs in myocardial diseases. Curr Heart Fail Rep. 2011;8(3):193-7.

8. Zhang Y, Wang Z, Gemeinhart RA. Progress in microRNA delivery. J Control Release. 2013:172(3):962-74.

9. Harraz MM, Eacker SM, Wang X, Dawson TM, Dawson VL. MicroRNA-223 is neuroprotective by targeting glutamate receptors. Proc Natl Acad Sci U S A. 2012;109(46):18962-7.

10. Moon J-m, Xu L, Giffard RG. Inhibition of microRNA-181 reduces forebrain ischemia-induced neuronal loss. J Cereb Blood Flow Metab. 2013;33(12): 1976-82.

11. Broughton BR, Reutens DC, Sobey CG. Apoptotic mechanisms after cerebral ischemia. Stroke. 2009;40(5):e331-9.

12. Liu W, Chen X, Zhang Y. Effects of microRNA-21 and microRNA-24 inhibitors on neuronal apoptosis in ischemic stroke. Am J Transl Res. 2016;8(7):317987.

13. Li P, Shen M, Gao F, Wu J, Zhang J, Teng F, et al. An antagomir to microRNA-106b-5p ameliorates cerebral ischemia and reperfusion injury in rats via inhibiting apoptosis and oxidative stress. Mol Neurobiol. 2017;54(4): 2901-21.

14. Liu X, Li F, Zhao S, Luo Y, Kang J, Zhao H, et al. MicroRNA-124-mediated regulation of inhibitory member of apoptosis-stimulating protein of p53 family in experimental stroke. Stroke. 2013;44(7):1973-80.

15. Sun L, Liu A, Zhang J, Ji W, Li Y, Yang X, et al. miR-23b improves cognitive impairments in traumatic brain injury by targeting ATG12-mediated neuronal autophagy. Behav. Brain Res. 2018;340:126-36.

16. Sabirzhanov B, Zhao Z, Stoica BA, Loane DJ, Wu J, Borroto C, et al. Downregulation of miR-23a and miR-27a following experimental traumatic brain injury induces neuronal cell death through activation of proapoptotic BCl-2 proteins. J Neurosci. 2014;34(30):10055-71.

17. Zhu H, Xie R, Liu X, Shou J, Gu W, Gu S, et al. MicroRNA-494 improves functional recovery and inhibits apoptosis by modulating PTEN/AKT/mTOR pathway in rats after spinal cord injury. Biomed Pharmacother. 2017;92:879-87.

18. Yi H, Huang Y, Yang F, Liu W, He S, Hu X. MicroRNA-182 aggravates cerebral ischemia injury by targeting inhibitory member of the ASPP family (iASPP). Arch Biochem Biophys. 2017;620:52-8.

19. Wang $P$, Liang $X$, Lu Y, Zhao X, Liang J. MicroRNA-93 Downregulation Ameliorates Cerebral Ischemic Injury Through the Nrf2/HO-1 Defense Pathway. Neurochem Res. 2016;41(10):2627-35.

20. Crack PJ, Taylor JM. Reactive oxygen species and the modulation of stroke. Free Radic Biol Med. 2005;38(11):1433-44.

21. Zhao H, Tao Z, Wang R, Liu P, Yan F, Li J, et al. MicroRNA-23a-3p attenuates oxidative stress injury in a mouse model of focal cerebral ischemiareperfusion. Brain Res. 2014;1592:65-72.

22. Jee MK, Jung JS, Choi Jl, Jang JA, Kang KS, Im YB, et al. MicroRNA 486 is a potentially novel target for the treatment of spinal cord injury. Brain J Neurol. 2012:135(Pt 4):1237-52

23. Liu da Z, Jickling GC, Ander BP, Hull H, Zhan X, Cox C, et al. Elevating microRNA-122 in blood improves outcomes after temporary middle cerebral artery occlusion in rats. J Cereb Blood Flow Metab. 2016;36(8):1374-83. 
24. Huang S, Ge X, Yu J, Han Z, Yin Z, Li Y, et al. Increased miR-124-3p in microglial exosomes following traumatic brain injury inhibits neuronal inflammation and contributes to neurite outgrowth via their transfer into neurons. FASEB J. 2018;32(1):512-28.

25. Li X-Q, Lv H-W, Wang Z-L, Tan W-F, Fang B, Ma H. MiR-27a ameliorates inflammatory damage to the blood-spinal cord barrier after spinal cord ischemia: reperfusion injury in rats by downregulating TICAM-2 of the TLR4 signaling pathway. J Neuroinflammation. 2015;12:25

26. Zhou HJ, Wang LQ, Xu QS, Fan ZX, Zhu Y, Jiang H, et al. Downregulation of miR-199b promotes the acute spinal cord injury through IKK $3-N F-K B$ signaling pathway activating microglial cells. Exp Cell Res. 2016;349(1):60-7.

27. Liu XS, Chopp M, Wang XL, Zhang L, Hozeska-Solgot A, Tang T, et al. MicroRNA-17-92 cluster mediates the proliferation and survival of neural progenitor cells after stroke. J Biol Chem. 2013;288(18):12478-88.

28. Jee MK, Jung JS, Im YB, Jung SJ, Kang SK. Silencing of miR2Oa is crucial for Ngn1mediated neuroprotection in injured spinal cord. Hum Gene Ther. 2012;23(5):508-20.

29. Li Y, Mao L, Gao Y, Baral S, Zhou Y, Hu B. MicroRNA-107 contributes to poststroke angiogenesis by targeting Dicer-1. Sci Rep. 2015;5(1):13316.

30. Ge X-T, Lei $P$, Wang H-C, Zhang A-L, Han Z-L, Chen X, et al. miR-21 improves the neurological outcome after traumatic brain injury in rats. Sci Rep. 2014;4(1):6718.

31. Liu D, Huang Y, Jia C, Li Y, Liang F, Fu Q. Administration of antagomir-223 inhibits apoptosis, promotes angiogenesis and functional recovery in rats with spinal cord injury. Cell Mol Neurobiol. 2015;35(4):483-91.

32. Ujigo S, Kamei N, Hadoush H, Fujioka Y, Miyaki S, Nakasa T, et al. Administration of microRNA-210 promotes spinal cord regeneration in mice. Spine. 2014;39(14):1099-107.

33. Sepramaniam S, Armugam A, Lim KY, Karolina DS, Swaminathan P, Tan JR, et al. MicroRNA 320a functions as a novel endogenous modulator of aquaporins 1 and 4 as well as a potential therapeutic target in cerebral ischemia. J Biol Chem. 2010;285(38):29223-30.

34. Sepramaniam S, Ying LK, Armugam A, Wintour EM, Jeyaseelan K. MicroRNA130a represses transcriptional activity of aquaporin 4 M1 promoter. J Biol Chem. 2012;287(15):12006-15.

35. Wei GJ, An G, Shi ZW, Wang KF, Guan Y, Wang YS, et al. Suppression of MicroRNA-383 Enhances Therapeutic Potential of Human BoneMarrow-Derived Mesenchymal Stem Cells in Treating Spinal Cord Injury via GDNF. Cell Physiol Biochem. 2017;41(4):1435-44.

36. Swarbrick S, Wragg N, Ghosh S, Stolzing A. Systematic review of miRNA as biomarkers in Alzheimer's disease. Mol Neurobiol. 2019;56(9):6156-67.

37. Angelucci F, Cechova K, Valis M, Kuca K, Zhang B, Hort J. MicroRNAs in Alzheimer's disease: diagnostic markers or therapeutic agents? Front Pharmacol. 2019;10:665.

38. Arshad AR, Sulaiman SA, Saperi AA, Jamal R, Mohamed Ibrahim N, Abdul Murad NA. MicroRNAs and target genes as biomarkers for the diagnosis of early onset of Parkinson disease. Front Mol Neurosci. 2017;10:352.

39. Ardashirova NS, Fedotova EY, Illarioshkin SN. The Role of MicroRNA in the Pathogenesis and Diagnostics of Parkinson's Disease. Neurochem J. 2020;14(2):12732.

40. Goh SY, Chao YX, Dheen ST, Tan E-K, Tay SS-W. Role of microRNAs in Parkinson's Disease. Int J Mol Sci. 2019;20(22):5649.

41. Xie Y, Chen Y. microRNAs: Emerging Targets Regulating Oxidative Stress in the Models of Parkinson's Disease. Front Neurosci. 2016;10:298.

42. Slota JA, Booth SA. MicroRNAs in neuroinflammation: implications in disease pathogenesis, biomarker discovery and therapeutic applications. Noncoding RNA. 2019;5(2):35

43. Yao L, Ye Y, Mao H, Lu F, He X, Lu G, et al. MicroRNA-124 regulates the expression of MEKK3 in the inflammatory pathogenesis of Parkinson's disease. J Neuroinflammation. 2018;15(1):13.

44. Tripathi AK, Tiwari SK, Mishra P, Jain M. Emerging role of microRNAs in cerebral stroke pathophysiology. In: Patnaik R, Tripathi AK, Dwivedi A, editors. Advancement in the pathophysiology of cerebral stroke. Singapore: Springer Singapore; 2019. p. 123-37.

45. Tan KS, Armugam A, Sepramaniam S, Lim KY, Setyowati KD, Wang CW, et al. Expression profile of MicroRNAs in young stroke patients. PLoS One. 2009; 4(11):e7689-e

46. Jeyaseelan K, Lim KY, Armugam A. MicroRNA expression in the blood and brain of rats subjected to transient focal ischemia by middle cerebral artery occlusion. Stroke. 2008;39(3):959-66.
47. Vasudeva K, Munshi A. miRNA dysregulation in ischaemic stroke: Focus on diagnosis, prognosis, therapeutic and protective biomarkers. Eur J Neurosci. 2020;52(6):3610-27.

48. Bansal A, Prathap R, Gupta S, Chaurasia A, Chaudhary P. Role of microRNAs in stroke recovery. J Family Med Prim Care. 2019;8(6):1850-4.

49. Brennan GP, Henshall DC. MicroRNAs as regulators of brain function and targets for treatment of epilepsy. Nat Rev Neurol. 2020;16(9):506-19.

50. Bauer S, Schütz V, Strzelczyk A, Rosenow F. Is there a role for microRNAs in epilepsy diagnostics? Expert Rev Mol Diagn. 2020;20(7):693-701.

51. Brennan GP, Henshall DC. microRNAs in the pathophysiology of epilepsy. Neurosci. Lett. 2018:667:47-52.

52. Yuan J, Huang H, Zhou X, Liu X, Ou S, Xu T, et al. MicroRNA-132 Interact with p250GAP/Cdc42 pathway in the hippocampal neuronal culture model of acquired epilepsy and associated with epileptogenesis process. Neural Plast. 2016;2016:5108489.

53. Hu K, Zhang C, Long L, Long X, Feng L, Li Y, et al. Expression profile of microRNAs in rat hippocampus following lithium-pilocarpine-induced status epilepticus. Neurosci Lett. 2011;488(3):252-7.

54. McCoy CE. miR-155 dysregulation and therapeutic intervention in multiple sclerosis. Adv Exp Med Biol. 2017;1024:111-31.

55. Piket E, Zheleznyakova GY, Kular L, Jagodic M. Small non-coding RNAs as important players, biomarkers and therapeutic targets in multiple sclerosis: a comprehensive overview. J Autoimmun. 2019;101:17-25.

56. Karnell FG, Lin D, Motley S, Duhen T, Lim N, Campbell DJ, et al. Reconstitution of immune cell populations in multiple sclerosis patients after autologous stem cell transplantation. Clin Exp Immunol. 2017;189(3): 268-78.

57. Waschbisch A, Atiya M, Linker RA, Potapov S, Schwab S, Derfuss T. Glatiramer acetate treatment normalizes deregulated microRNA expression in relapsing remitting multiple sclerosis. PLoS One. 2011;6(9):e24604-e.

58. Amoruso A, Blonda M, Gironi M, Grasso R, Di Francescantonio V, Scaroni F, et al. Immune and central nervous system-related miRNAs expression profiling in monocytes of multiple sclerosis patients. Sci Rep. 2020;10(1): 6125.

59. Mohammed EM. Environmental influencers, microRNA, and multiple sclerosis. J Cent Nerv Syst Dis. 2020;12:1179573519894955.

60. Sheedy FJ. Turning 21: induction of miR-21 as a key switch in the inflammatory response. Front Immunol. 2015;6:19.

61. Zhou Z, Xiong $H$, Xie F, Wu Z, Feng Y. A Meta-analytic review of the value of miRNA for multiple sclerosis diagnosis. Front Neurol. 2020;11:132.

62. Wu T, Chen G. miRNAs participate in MS pathological processes and its therapeutic response. Mediat Inflamm. 2016;2016:4578230.

63. Koval ED, Shaner C, Zhang P, du Maine X, Fischer K, Tay J, et al. Method for widespread microRNA-155 inhibition prolongs survival in ALS-model mice. Hum Mol Genet. 2013;22(20):4127-35.

64. Li C, Wei Q, Gu X, Chen Y, Chen X, Cao B, et al. Decreased glycogenolysis by miR-338-3p promotes regional glycogen accumulation within the spinal cord of amyotrophic lateral sclerosis mice. Front Mol Neurosci. 2019;12:114.

65. Rizzuti M, Filosa G, Melzi V, Calandriello L, Dioni L, Bollati V, et al. MicroRNA expression analysis identifies a subset of downregulated miRNAs in ALS motor neuron progenitors. Sci Rep. 2018;8(1):10105.

66. Yamakuchi M, Ferlito M, Lowenstein CJ. miR-34a repression of SIRT1 regulates apoptosis. Proc Natl Acad Sci. 2008;105(36):13421.

67. Emde A, Eitan C, Liou LL, Libby RT, Rivkin N, Magen I, et al. Dysregulated miRNA biogenesis downstream of cellular stress and ALS-causing mutations: a new mechanism for ALS. EMBO J. 2015;34(21):2633-51.

68. Bañez-Coronel M, Porta S, Kagerbauer B, Mateu-Huertas E, Pantano L, Ferrer l, et al. A pathogenic mechanism in Huntington's disease involves small CAG-repeated RNAs with neurotoxic activity. PLoS Genet. 2012;8(2): e1002481.

69. Johnson R, Zuccato C, Belyaev ND, Guest DJ, Cattaneo E, Buckley NJ. A microRNA-based gene dysregulation pathway in Huntington's disease. Neurobiol Dis. 2008;29(3):438-45.

70. Packer AN, Xing Y, Harper SQ, Jones L, Davidson BL. The bifunctional microRNA miR-9/miR-9* regulates REST and COREST and is downregulated in Huntington's disease. J Neurosci. 2008;28(53):14341-6.

71. Lee ST, Chu K, Im WS, Yoon HJ, Im JY, Park JE, et al. Altered microRNA regulation in Huntington's disease models. Exp Neurol. 2011;227(1):172-9.

72. Wang L, Zhao C, Wu S, Xiao G, Zhuge X, Lei P, et al. Hydrogen Gas Treatment Improves the Neurological Outcome After Traumatic Brain Injury Via Increasing miR-21 Expression. Shock. 2018;50(3):308-15. 
73. Song J, Li N, Xia Y, Gao Z, Zou SF, Yan YH, et al. Arctigenin confers neuroprotection against mechanical trauma injury in human neuroblastoma SH-SY5Y cells by regulating miRNA-16 and miRNA-199a expression to alleviate inflammation. J Mol Neurosci. 2016:60(1):115-29.

74. Li Z, Wang S, Li W, Yuan H. Ferulic acid improves functional recovery after acute spinal cord injury in rats by inducing hypoxia to inhibit microRNA-590 and elevate vascular endothelial growth factor expressions. Front Mol Neurosci. 2017;10:183.

75. Yang Q, Yang K, Li AY. Trimetazidine protects against hypoxia-reperfusioninduced cardiomyocyte apoptosis by increasing microRNA-21 expression. Int J Clin Exp Pathol. 2015:8(4):3735-41.

76. Wen Y, Zhang X, Dong L, Zhao J, Zhang C, Zhu C. Acetylbritannilactone modulates microRNA-155-mediated inflammatory response in ischemic cerebral tissues. Mol Med. 2015;21(1):197-209.

77. Li L, Jiang HK, Li YP, Guo YP. Hydrogen sulfide protects spinal cord and induces autophagy via miR-30c in a rat model of spinal cord ischemiareperfusion injury. J Biomed Sci. 2015:22(1):50.

78. Dong Y-F, Chen Z-Z, Zhao Z, Yang D-D, Yan H, Ji J, et al. Potential role of microRNA-7 in the anti-neuroinflammation effects of nicorandil in astrocytes induced by oxygen-glucose deprivation. J Neuroinflammation. 2016;13(1):60.

\section{Publisher's Note}

Springer Nature remains neutral with regard to jurisdictional claims in published maps and institutional affiliations.

\section{Submit your manuscript to a SpringerOpen ${ }^{\circ}$ journal and benefit from:}

- Convenient online submission

- Rigorous peer review

- Open access: articles freely available online

- High visibility within the field

- Retaining the copyright to your article

Submit your next manuscript at $\boldsymbol{\nabla}$ springeropen.com 